\title{
The self-enrichment of galactic halo globular clusters
}

\section{The mass-metallicity relation}

\author{
G. Parmentier ${ }^{1,2, \star}$ and G. Gilmore ${ }^{2}$ \\ 1 Institute of Astrophysics and Geophysics, University of Liège, avenue de Cointe 5, 4000 Liège, Belgium \\ 2 Institute of Astronomy, Madingley Road, Cambridge CB3 OHA, UK \\ Received 10 July 2001 / Accepted 16 August 2001

\begin{abstract}
We discuss the existence of a mass-metallicity relation among galactic halo globular clusters. The lack of any luminosity-metallicity correlation in globular cluster systems has been used as an argument against self-enrichment models of cluster formation. We show that such a relation is statistically present among the galactic Old Halo globulars. This observational correlation implies that the least massive old clusters are the most metal-rich. This is in contradiction with the idea that, if globular clusters were self-enriched systems, the most metal-rich clusters would also be the most massive ones. We further show that this anti-correlation is as predicted by self-enrichment models.
\end{abstract}

Key words. Galaxy: evolution - Galaxy: formation - globular clusters: general - Galaxy: halo

\section{Introduction}

Galactic Globular Clusters are among the very first bound structures that formed in what later became the Galaxy. As such, individual globular clusters and the Globular Cluster System provide us with important insights concerning the age, the formation and the early evolution of the Galaxy.

This paper is the third of a series devoted to the study of a formation scenario of galactic halo globular clusters, namely the self-enrichment hypothesis, which develops the Fall \& Rees (1985) cluster formation model. The model is detailed in Parmentier et al. (1999) (hereafter Paper I) and a summary is provided in Parmentier et al. (2000) (hereafter Paper II), Sect. 2.

The model assumes that primordial cold clouds embedded in a hot and diffuse protogalactic background (Fall \& Rees 1985) are the gaseous progenitors of galactic halo globular clusters, that is, this model assumes baryon assembly predates star formation. Our model explores the ability of these proto-globular cluster clouds to retain the ejecta of a first generation of zero-metal abundance stars, born in the central regions of the clouds. When the massive stars explode as type II supernovae, they chemically enrich the surrounding pristine interstellar medium and trigger the expansion of a supershell in which a second generation of nonzero-metal abundance stars may form. The aim of a self-enrichment scenario is therefore to explain both the

Send offprint requests to: G. Parmentier,

e-mail: parmentier@astro.ulg.ac.be

* Visiting Marie Curie Fellow at the Institute of Astronomy, Cambridge University. formation of a globular cluster and the origin of its metal content.

One of the key parameters of this class of model is the external pressure exerted by the hot protogalactic background on the proto-clusters. The higher the pressure is (i.e. the deeper the proto-cluster is located in the protoGalaxy in the simplest implementation of the model), the smaller its mass is, the higher its metallicity will be (see Table 1 of Paper I).

An in-depth discussion of the ensuing Galactic metallicity gradient is presented in Paper II. We show that, when combined with a pressure profile scaling as $P_{\mathrm{h}} \propto$ $D^{-2}$, where $P_{\mathrm{h}}$ is the hot protogalactic background pressure and $D$ is the galactocentric distance, the model is consistent with the metallicity gradient observed for the Old Halo globular cluster system.

There are three aspects of globular cluster formation which self-enrichment models must specifically address. The disruptive effects of supernovae, and the internal chemical homogeneity are discussed in Paper I. This paper considers the third aspect, the extent to which a mass(luminosity)-metallicity relation is expected and observed. The Galactic globular cluster system is used as a specific example.

\section{Self-enrichment and a mass-metallicity relation}

The lack of any obvious correlation, in any globular cluster system, between the mass (or the luminosity) and the metallicity of individual globulars is often used as an argument against the self-enrichment hypothesis. Indeed, were one to assume that gravitational potential gradients 


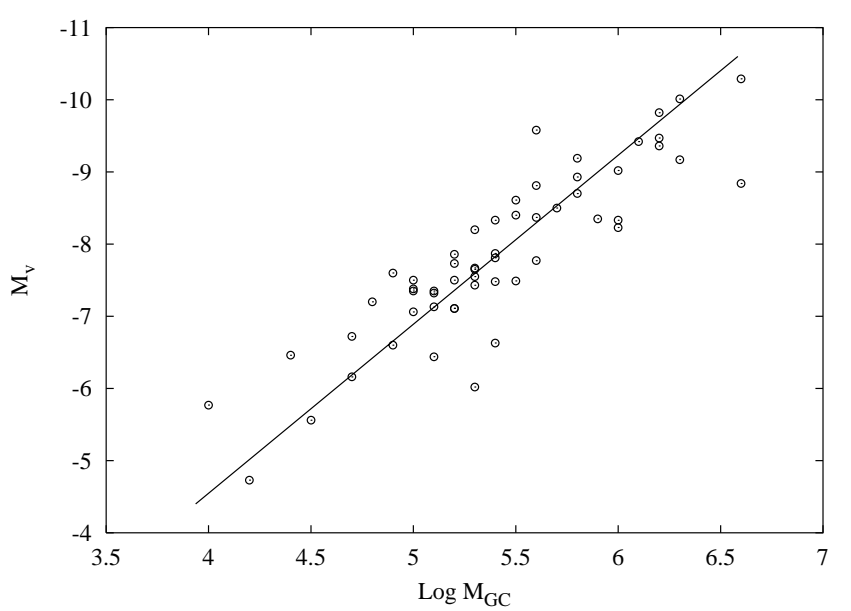

Fig. 1. Mass-luminosity relation for the 56 globular clusters of the Pryor \& Meylan (1993) compilation and the corresponding least-squares fit.

dominated mass loss, the most massive objects would be better able to retain their metal-enriched supernova ejecta, so that metal abundance should increase with cluster mass in case of self-enrichment. Before adressing the discussion of a luminosity-metallicity relation, we would like to dismiss this idea that more massive clusters would be more metal-rich in the case of self-enrichment. If a more massive object is indeed better able to retain more supernova ejecta, this larger amount of metallic ejecta is mixed with a larger amount of gas. Therefore, no firm conclusion can be drawn concerning the resulting metal abundance (or metallicity), i.e. the ratio of the two increased quantities. It is the fractional efficiency of gas retention which is important. Most importantly, though, mass loss in this class of models is determined by external gas pressure and not by the pressure equivalent of the gravitational potential gradient. This means that the absence of a mass-metallicity relation, in the sense that the most massive globulars would also be the most metal-rich (e.g. McLaughlin 1997; Barmby et al. 2000), can not be considered as evidence against the self-enrichment hypothesis. In sharp opposition with these statements, the selfenrichment model we develop foresees a mass-metallicity relationship in the sense that the most metal-rich protoglobular clusters are the least massive ones.

Unlike globular clusters, dwarf galaxies exhibit welldefined correlations between luminosity and metallicity (e.g. Gilmore 2000; Mateo 2000) such that the dimmest ones are the most metal weak. The standard explanation for this correlation being self-enrichment in the presence of galactic winds which are limited by gravitational potential gradients (Dekel \& Silk 1986), Djorgovski \& Meylan (1994) conclude that globular clusters cannot be self-enriched systems. However, Dekel \& Silk (1986) point out that the dwarf galaxy observed luminositymetallicity relation can be successfully explained only if the gaseous proto-galaxies are embedded within dominant halos of dark matter. While there is indeed clear evidence of the presence of such halos around dwarf galaxies

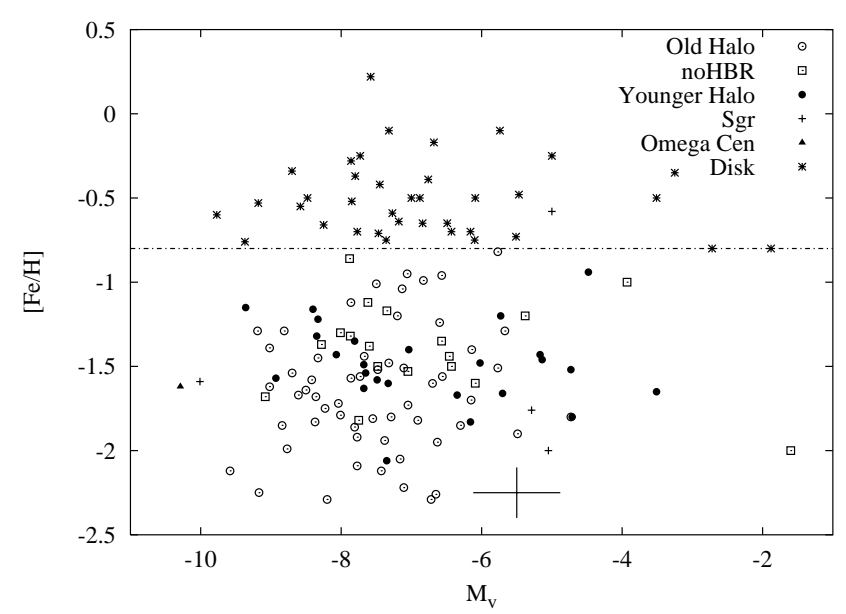

Fig. 2. Metallicity-luminosity diagram for the whole galactic globular cluster sytem. The dashed line at $[\mathrm{Fe} / \mathrm{H}]=-0.8$ represents the generally assumed metallicity limit between the halo and the bulge/disk subsystems. The different types of globulars are marked by different symbols. Old Halo and Younger Halo clusters are respectively represented by open and full circles. The crosses label the Sgr globulars. The open squares ("noHBR" group) stand for the halo globular clusters for which the horizontal brach morphology index is not given in Harris (1996). The full triangle and the asterisks respectively represent $\omega$ Cen and the bulge/disk clusters.

(Mateo 1996), this is not the case for globular clusters (Moore 1996; Meylan \& Heggie 1997). Therefore, the Dekel \& Silk model, built for dwarf galaxies, can certainly be not extrapolated to globular clusters. Moreover, dwarf galaxies and GCs have undergone very different star formation histories: their respective star formation rate and duration differ by, at least, an order of magnitude (Gilmore 2000). Dwarf galaxies also exhibit metallicity spreads, often larger than 1 dex (Mateo 2000), in marked contrast with the chemical homogeneity of globular clusters. Considering these many differences, the comparison between globular clusters and dwarf galaxies therefore appears irrelevant.

In searching for a luminosity-metallicity relation in the Galactic globular cluster system, it should be kept in mind that, while the observed quantity is the luminosity, the physical quantity of interest is the mass. Figure 1 represents the relation between the mass and the absolute visual magnitude for the 56 globular clusters of the Pryor \& Meylan (1993) mass compilation. The $M_{v}$ values come from the McMaster Catalogue (Harris 1996, updated 1999). The scatter superimposed on the correlation, of the order of $\sigma_{M_{v}} \simeq 0.6$, is equivalently the variations of the mass-to-light ratio from cluster to cluster. This ranges from $\sim 1$ to $\sim 4$ (Pryor \& Meylan 1993) and reflects possible differences in the initial mass function and the dynamical evolution of the clusters ${ }^{1}$. Therefore, any

\footnotetext{
${ }^{1}$ Part of the scatter also originates in the uncertainties of globular cluster mass computation, since it relies on model assumptions such as the isotropy of velocity distibution and a maxwellian distribution of velocities.
} 
mass-metallicity correlation will be, at least partly, washed out in the corresponding $M_{v}-[\mathrm{Fe} / \mathrm{H}]$ plot. This effect is illustrated in Fig. 2, the metallicity-luminosity diagram for the whole globular cluster system (the $[\mathrm{Fe} / \mathrm{H}]$ values are taken from the McMaster Catalogue). Also plotted are the corresponding error bars in $[\mathrm{Fe} / \mathrm{H}], \pm 0.15 \mathrm{dex}$ (King 1999), and in $M_{v}, \pm 0.6$ dex from Fig. 1, if the latter is considered to be a mass indicator. The size of the $M_{v}$ errorbars (reflecting the different luminosities that a globular cluster with a given mass but varying mass-to-light ratios may exhibit) is clearly not negligeable compared to the size of the observed distribution, the dispersion of the best-fitting gaussian to the galactic globular cluster luminosity function being $\simeq 1.2$ (Harris 1991).

Unfortunately, determination of the physical quantity of interest, i.e. the relative masses of the globular clusters at their formation, is still uncertain at least by a factor 2 (Meylan 2000). For instance, the use of single-mass King models is a simplification which tends to underestimate cluster mass (Ashman \& Zepf 1998; Mermilliod 2000). Therefore, one of the key points in the search for a mass-metallicity correlation is to use an homogeneous set of globular cluster masses in order to limit additional scatter in the $\left(\log M_{\mathrm{GC}},[\mathrm{Fe} / \mathrm{H}]\right)$ plot. We use the globular cluster mass compilation computed by Pryor \& Meylan (1993): this compilation is the most complete set of globular cluster masses computed with an internally consistent family of multi-component King-Michie models.

Another source of scatter in the luminosity(mass)metallicity plot is introduced by the various origins of the Galactic globulars. Indeed, evidence has now accumulated that the Galactic globular cluster system does not consist of globular clusters with a single origin. While the majority of globular clusters in the halo are old, with a remarkably small age spread (Rosenberg et al. 1999), there is a small subset, particularly among the more metal-rich clusters, with inferred ages of several Gyr younger than the dominant old population. These younger globular clusters are either clusters being/having been accreted by the Galaxy recently or metal-rich clusters associated with the bulge/disk subsystem. These clusters being significantly younger, their formation is not expected to be taken into account by our self-enrichment model, which deals with globular clusters whose gaseous progenitors have a primordial composition.

The age spread highlighted by Rosenberg et al. (1999) also confirms the globular cluster system subdivisions early suggested by Zinn $(1985,1993)$. From the point of view of the metallicity distribution, the Galactic globular cluster system is composed of two subpopulations, a metal-poor halo group and a metal-rich, centrally concentrated, bulge (or disk) group (Zinn 1985). Furthermore, the halo subsystem itself includes an Old Halo, made of globular clusters perhaps born in situ, during the collapse of the protogalactic cloud, and a Younger Halo likely made of globulars later stripped from neighbouring dwarf galaxies (see Paper II for a review of these evidences).

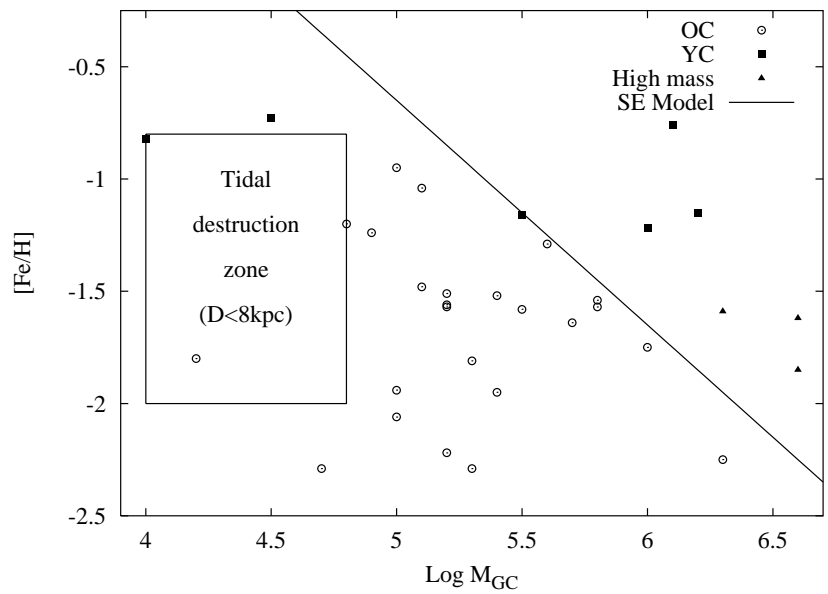

Fig. 3. $[\mathrm{Fe} / \mathrm{H}]$ vs. $\log M_{\mathrm{GC}}$ diagram for the globular clusters studied by Rosenberg et al. (1999). The old (OC) and the younger (YC) globular clusters exhibit different distributions in the diagram relatively to the boundary foreseen by the selfenrichment model. Also shown is the tidal destruction zone for the less massive halo globular clusters located inside the solar circle.

Since our self-enrichment model deals with globular clusters whose progenitors were embedded in the hot phase of the protogalactic cloud and whose gaseous material was pristine, it is not expected to apply to the Younger Halo group, the presumed accreted component of the halo, nor to the bulge clusters. Thus, in what follows, we focus either on the coeval and old sample of Rosenberg et al. (1999) or on the Old Halo defined by Zinn.

\section{Comparison of the model with the observations}

Our self-enrichment model suggests the existence of an anti-correlation between the mass $M$ of a proto-cluster and the metallicity $[\mathrm{Fe} / \mathrm{H}]$ reached at the end of the selfenrichment process, in the sense that the least massive proto-clusters create the most metal-rich globular clusters (see Table 1 of Paper I):

$[\mathrm{Fe} / \mathrm{H}]=4.3-\log M$.

However, this (anti-)correlation applies to the gaseous progenitors; the mass-metallicity relation observed among the studied sample of clusters, if any, should only be a relic of Eq. (1). For instance, the -1 slope will be conserved only if there is a universal and constant star formation efficiency for the second stellar generation which forms the majority of the stars from the chemically enriched gas swept up from the first generation supernovae. Since the mass $M$ of a gaseous progenitor is an upper limit for the mass $M_{\mathrm{GC}}$ of the globular cluster formed, Eq. (1) delimits a permitted area in the $\left(\log M_{\mathrm{GC}},[\mathrm{Fe} / \mathrm{H}]\right)$ plot: all the data should be located to the left of Eq. (1) (plain curve in Figs. 3 and 5). Figure 3 represents Eq. (1) together with the globular clusters for which the age and the mass are respectively provided in Rosenberg et al. (1999) and Pryor \& Meylan (1993). Obviously, the two Rosenberg et al. (1999) 


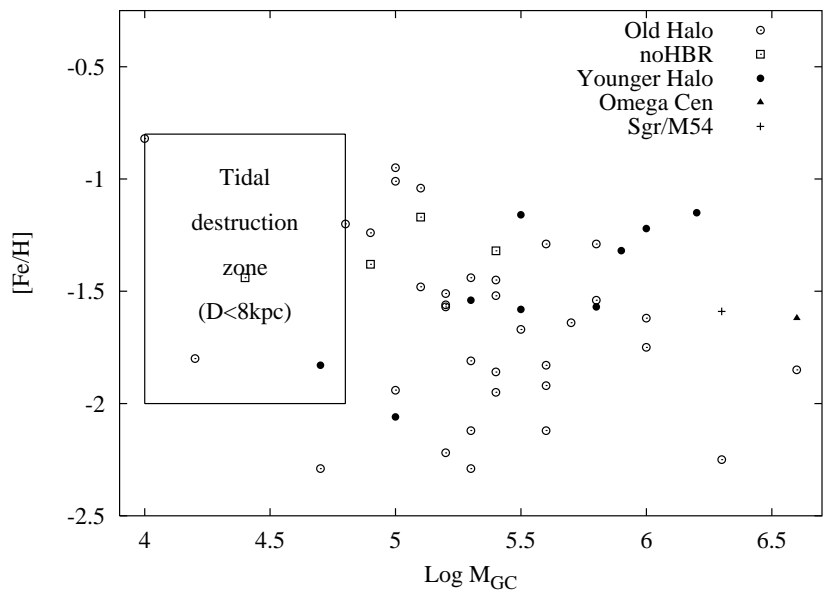

Fig. 4. $[\mathrm{Fe} / \mathrm{H}]$ vs. $\log M_{\mathrm{GC}}$ plot including Old Halo and Younger Halo subgroups (49 globular clusters).

groups (old clusters: open symbols; younger clusters: filled symbols) behave in a different way compared to our selfenrichment mass-metallicity relation. While the old, coeval and metal-poor GCs are all located in the permitted area of the plot, i.e. their mass-metallicity diagram is consistent with the self-enrichment of primordial gaseous progenitors, half of the young clusters, either presumed accreted or belonging to the bulge subsystem, are located in the forbidden area of the diagram, i.e. as expected, their formation cannot be accounted for by the self-enrichment model. In Fig. 3, we also represent three of the most massive globulars (filled triangles), namely $\omega$ Cen, M 54 and NGC 5824. Their location in the forbidden part of the plot points to a different star formation history. This is not surprising since, at least in the case of $\omega$ Cen and M 54, an intrinsic abundance spread is seen. M 54 is of course a member of the Sagittarius dwarf spheroidal galaxy, and is not (yet) a Galactic globular cluster.

Figure 3 is also clearly depleted in low-mass globular clusters $\left(\log M_{\mathrm{GC}}<4.8\right)$. However, at a galactocentric distance smaller than $8 \mathrm{kpc}$, these low-mass clusters are not expected to survive more than a Hubble time (see the "survival triangle" in the mass vs half-mass radius diagram defined by Gnedin \& Ostriker 1996, their Fig. 20a). The vast majority of the globular clusters located at these galactocentric distances, i.e. $\log D<8 \mathrm{kpc}$, exhibit a metallicity higher than $[\mathrm{Fe} / \mathrm{H}]=-2$. The depletion zone, represented by the box in Figs. 3 and 4, is therefore not surprising and corresponds to the tidal destruction of these low-mass clusters. The globular clusters used in our Paper are therefore no more than a surviving sample. The distance a given cluster lies to lower masses from the model upper bound is, to first order, a measure of the star formation efficiency of cluster formation. A "typical" surviving cluster lies a factor of order 5 below the bound, suggesting an efficiency factor of order $20 \%$. As noted above, however, lower mass clusters will have preferentially failed to survive until today, so that this value is an upper limit. Star formation efficiencies in the range from a few to a few tens of percent seem appropriate for

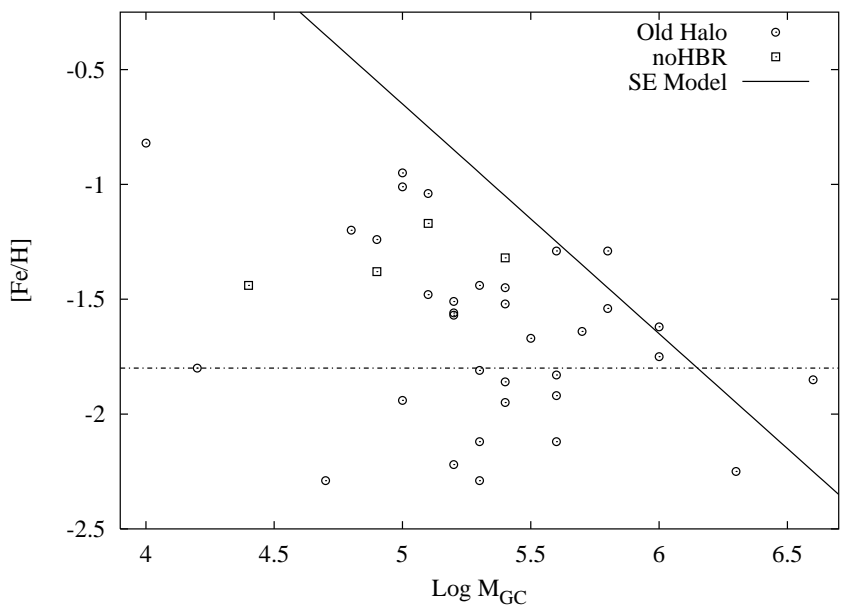

Fig. 5. $[\mathrm{Fe} / \mathrm{H}]$ vs. $\log M_{\mathrm{GC}}$ plot for the Old Halo subgroup (37 globular clusters). The self-enrichment model (plain curve) defines a permitted area (left part of the plot) in which most of the observational points are located. The correlation between the globular cluster masses and their metallicities is particularly striking for $[\mathrm{Fe} / \mathrm{H}]>-1.8$.

most clusters. Only the few percent of clusters which are the most massive require star formation efficiencies in excess of unity, and so are inconsistent with this formation model. Interestingly, these very massive clusters are those which show internal abundance spreads, which are themselves direct evidence for self-enrichment during cluster formation.

In order to increase our sample and to look for a surviving correlation between the mass and the metallicity, we also consider the Old Halo subgroup (Zinn 1993). As for the metallicity gradient (see Paper II), an Old Halo/Younger Halo separation is fruitful. Figure 4 shows a plot of $[\mathrm{Fe} / \mathrm{H}]$ versus mass for the 49 halo globular clusters whose mass has been computed by Pryor \& Meylan (1993): there is no correlation between the mass and the metallicity, the linear Pearson correlation coefficient being -0.15 .

Considering the Old Halo group only (Fig. 5), as stated in the previous Section, a weak correlation between the logarithm of the mass of the globular clusters and their metallicity emerges. The linear Pearson correlation coefficient improves to a value of -0.35 , with a corresponding probability of correlation of $96.92 \%$. Moreover, most of the Old Halo globulars are located in the permitted area of the plot ${ }^{2}$.

\footnotetext{
${ }^{2}$ If we consider the Old Halo clusters with a metallicity larger than -1.8 , the increase of the metallicity with decreasing mass is strengthened: the linear Pearson correlation coefficient is -0.64 corresponding to a probability of correlation of $99.93 \%$. One can question about the validity of dividing the Old Halo group at a metallicity of -1.8 . According to Zinn (1996) and Dinescu et al. (1999), the Old Halo group with $[\mathrm{Fe} / \mathrm{H}]>-1.8$ is kinematically distinguishable from the Old Halo group with $[\mathrm{Fe} / \mathrm{H}]<-1.8$ (their Metal-Poor component). However, more kinematical data are needed to clarify this tentative point.
} 


\section{Conclusions}

We present the mass-metallicity relation foreseen by our self-enrichment model. At first glance, such a globular cluster formation scenario is disproven by the lack of any obvious correlation between the luminosity and the metallicity of globular clusters, as claimed by previous authors (e.g. Djorgovski \& Meylan 1994; Ashman \& Zepf 1998). However, we stress here that there are numerous sources of scatter between the theoretical (mass, $[\mathrm{Fe} / \mathrm{H}]$ ) relation, applying to the gaseous progenitors of globular clusters, and the observed (luminosity, $[\mathrm{Fe} / \mathrm{H}]$ ) plot, applying to globular clusters. These scatter sources are, for instance, the star formation efficiency with which the globular cluster stars form out of the proto-globular cluster cloud, the mass losses undergone by globular clusters with time (see the tidal tails exhibited by some clusters), the variations in the mass-to-light ratio from one globular cluster to another. We also caution that the search for a massmetallicity correlation should be restricted to a given globular cluster subpopulation, namely the Old Halo group. Despite the numerous sources of scatter, the globulars of this group are characterized by a boundary in the massmetallicity diagram and by a correlation in the sense expected by simple pressure-bounded self-enrichment models. Self-enrichment models remain a viable hypothesis for galactic halo globular cluster formation.

Acknowledgements. This research was supported partly by the European Community under grant HPMT-CT-2000-00132 and partly by contracts Pôle d'Attraction Interuniversitaire P4/05 (SSTC, Belgium) and FRFC F6/15-OL-F63 (FNRS, Belgium).

\section{References}

Ashman, K. M., \& Zepf, S. E. 1998, Globular Cluster Systems, Cambridge Astrophys. Ser.

Barmby, P., Huchra, J. P., Brodie, J. P., et al. 2000, AJ, 119, 727

Dekel, A., \& Silk, J. 1986, ApJ, 303, 39
Dinescu, D. I., Girard, T. M., \& Van Altena, W. F. 1999, AJ, 117,1792

Djorgovski, S., \& Meylan, G. 1994, AJ, 108, 1292

Fall, S. M., \& Rees, M. J. 1985, ApJ, 298, 18

Gilmore, G. 2000, in Galaxy Disks and Disk Galaxies, ed. J. G. Funes, \& E. M. Corsini, ASP Conf. Ser. 230, 3

Gnedin, O. Y., \& Ostriker, J. P. 1997, ApJ, 474, 223

Harris, W. E. 1991, ARA\&A, 29, 543

Harris, W. E. 1996, AJ, 112, 1487

King, I. R. 1999, in Globular Clusters, ed. C. Martinez Roger, I. Pérez Fournon, \& F. Sanchez (Cambridge University Press), 1

Mateo, M. 1996, in Formation of the Galactic Halo... Inside and Out, ed. H. Morrison, \& A. Sarajedini, ASP Conf. Ser., 92, 434

Mateo, M. 2000, in The First Stars (Proceedings of the MPA/ESO Workshop held at Garching, Germany, 4-6 August 1999), ed. A. Weiss, T. G. Abel, \& V. Hill (Springer), 283

McLaughlin, D. E. 1997, Ph.D. Thesis, McMaster University (Canada)

Mermilliod, J.-C. 2000, in Massive Stellar Clusters, ed. A. Lancon, \& C. M. Boily (San Fransisco), ASP Conf. Ser., 211,43

Meylan, G. 2000a, in The Galactic Halo: from globular clusters to field stars, ed. A. Noels, P. Magain, D. Caro, et al., 35th Liège International Astrophys. Colloq., 543

Meylan, G., \& Heggie, D. C. 1997, A\&AR, 8, 1

Moore, B. 1996, ApJ, 461, L13

Parmentier, G., Jehin, E., Magain, P., et al. 1999, A\&A, 352, 138

Parmentier, G., Jehin, E., Magain, P., Noels, A., \& Thoul, A. 2000, A\&A, 363, 526

Pryor, T., \& Meylan, G. 1993, in Structure and Dynamics of Globular Clusters, ed. S. G. Djorgovski, \& G. Meylan (San Fransisco), ASP Conf. Ser., 50, 357

Rosenberg, A., Saviane, I., Piotto, G., \& Aparicio, A. 1999, AJ, 118, 2306

Zinn, R. 1985, ApJ, 293, 424

Zinn, R. 1993, in ASP Conf. Ser. 48, The Globular ClusterGalaxy Connection, ed. G.H. Smith, \& J.P. Brodie (San Fransisco), 38

Zinn, R. 1996, in Formation of the Galactic Halo... Inside and Out, ed. H. Morrison, \& A. Sarajedini (San Fransisco), ASP Conf. Ser., 92, 38 